\title{
Clinical features of pediatric post-acute COVID-19: a descriptive retrospective follow-up study
}

\author{
Liene Smane ${ }^{1,2^{*}}$, leva Roge ${ }^{1}, Z^{2}$ anda Pucuka ${ }^{1,2}$ and Jana Pavare ${ }^{1,2}$
}

\begin{abstract}
To date, information on COVID-19 long-term post-recovery sequelae in children and adolescents remains scarce. A retrospective descriptive cohort study was performed by collecting data on 92 patients (age $\leq 18$ years). All were evaluated during a face-to-face visit following a specially designed post-COVID-19 symptom assessment protocol at the following stage: 1-3 months after COVID-19 onset. Among the 92 children, 45 (49\%) were completely free of any COVID-19-related symptoms, while 47 (51\%) reported persistence of at least one symptom, in particular tiredness, loss of taste and/or smell and headaches. The most common post-acute COVID-19 clinical features were noted in children aged between 10 and 18 years. A detailed multidisciplinary follow-up of patients with COVID-19 seems relevant, whatever the severity of the symptoms.
\end{abstract}

Keywords: SARS-CoV-2, Post-acute COVID-19, Pediatrics

To the Editor,

Severe acute respiratory syndrome coronavirus 2 (SARS-CoV-2) infection causes a spectrum of characteristics ranging from asymptomatic seroconversion to severe coronavirus cases, sometimes with prolonged symptoms [1]. Current data shows that prolonged symptom duration is common not only in adults with severe and non-severe coronavirus disease 2019 (COVID-19), but also among children with COVID-19 [2-7]. Postacute COVID-19 clinical features vary widely. The most commonly reported persistent symptoms after recovery from COVID-19 among the symptomatic outpatients were insomnia, fatigue, coughing, dyspnea, loss of taste and/or smell and headaches [3, 5-7]. As noted by Hoang et al., SARS-CoV-2 infection in children has a milder clinical course commonly characterised by fever, cough, rhinorrhea and myalgia/fatigue [8]. This contrasts with

\footnotetext{
* Correspondence: Liene.Smane@rsu.lv

'Children's Clinical University Hospital, Riga, Latvia

${ }^{2}$ Department of Paediatrics, Riga Stradins University, Riga, Latvia
}

adults, characterising a return to baseline health among children with milder COVID-19, although the clinical course is not sufficiently described.

Children's Clinical University Hospital in Riga established a post-acute outpatient follow-up service for individuals who had recovered from COVID-19. Before the start of December 2020, 3509 children were diagnosed with SARS-CoV-2 infection, representing approximately $8.6 \%$ of all COVID-19 patients in Latvia. We conducted a retrospective cohort study, including 92 COVID-19 disease outpatients (age $\leq 18$ years) and their caregivers (parents or legal family representatives) from March 12, 2020 (the date COVID-19 was declared a state of emergency in Latvia), to December 1, 2020. A real-time polymerase chain reaction (RT-PCR) for SARS-CoV-2 was used, and the study included children with a negative test result. Patients were tested systematically for viral co-infections. Caregivers had to provide written consent for this. To identify the long-term consequences of SARS-CoV-2 infection, all patients enrolled in the study were evaluated by two pediatricians in a 
Table 1 Characteristics of Symptomatic Children's COVID-19 Cases $(N=92)$

Characteristics
Median (interquartile range) age, years
Age group, No. (\%):
1-4 years
5-9years
10-14 years
15-18 years
Sex at birth, No. (\%):
Male
Female
Vaccination, No. (\%)
Seasonal influenza
Complete vaccination
Incomplete vaccination
Total baseline chronic medical conditions, No. (\%)
Bronchial asthma
Attention-deficit/hyperactivity disorder
Atopic dermatitis
Arthritis
Patent ductus arteriosus
Vegetative dystonia
Eongenital hypothyroidism
Overactive bladder

Exposure to the source of transmission, No. (\%)

Contact with COVID19 positive patients within the last 14 days

Contact with group COVID-19 cases

Contact with a person with fever / respiratory symptoms or epidemiological communities in the last 14 days

Contact with a person who does not know the COVID-19 test results within 14 days

Traveling in the last 14 days

Patients from a care center Value

$12(8-15)$

None

$10(11 \%)$

$21(23 \%)$

$35(38 \%)$

$26(28 \%)$

$56(61 \%)$

$36(39 \%)$

$12(13 \%)$

$85(92 \%)$

$12(13 \%)$

$18(20 \%)$

$7(8 \%)$

$3(3 \%)$

$2(2 \%)$

$1(1 \%)$

$1(1 \%)$

$1(1 \%)$

$1(1 \%)$

$1(1 \%)$

$1(1 \%)$

$66(72 \%)$

$35(38 \%)$

$22(24 \%)$

$18(20 \%)$

$17(18 \%)$

$2(2 \%)$

Acute COVID-19 symptoms, No. (\%)

Distribution of temperature, No. (\%)

$\begin{array}{ll}37.2-37.5^{\circ} \mathrm{C} & 36(39 \%) \\ \geq 37.5-38^{\circ} \mathrm{C} & 15(16 \%) \\ 38.1-39.0^{\circ} \mathrm{C} & 13(14 \%) \\ >39.0^{\circ} \mathrm{C} & 11(12 \%) \\ \text { Tiredness } & 42(46 \%) \\ \text { Headache } & 34(37 \%) \\ \text { Cough } & 30(33 \%) \\ \text { Rhinorrhea } & 29(32 \%) \\ \text { Sore throat } & 29(32 \%)\end{array}$

Table 1 Characteristics of Symptomatic Children's COVID-19 Cases $(N=92)$ (Continued)

\begin{tabular}{ll}
\hline Characteristics & Value \\
\hline Loss of taste and/or smell & $27(29 \%)$ \\
Stuffy nose & $26(28 \%)$ \\
Sneezing & $22(24 \%)$ \\
Diarrhea & $17(18 \%)$ \\
Myalgia & $13(14 \%)$ \\
Shortness of breath & $10(11 \%)$ \\
Drowsiness & $7(8 \%)$ \\
Decreased appetite & $5(5 \%)$ \\
Conjunctivitis & $5(5 \%)$ \\
Wheezing & $5(5 \%)$ \\
Vomiting & $4(4 \%)$ \\
Sensory disturbances & $4(4 \%)$ \\
Sweatiness & $2(2 \%)$ \\
Epistaxis & $2(2 \%)$ \\
Periorbital edema & $1(1 \%)$ \\
Seizures & $1(1 \%)$ \\
Post-acute CoviD-19 follow-up characteristics & \\
Days since symptoms onset, median (interquartile range) & $55(30-104)$ \\
Persistent symptoms, No. (\%) & \\
None & $45(49 \%)$ \\
1 & $18(19 \%)$ \\
2 & $9(10 \%)$ \\
\hline
\end{tabular}

face-to-face visit according to a specially designed postCOVID-19 symptom assessment protocol (supplementary material) developed based on previously published literature about Long COVID in adults [2, 3, 9, 10]. Post-COVID-19 evaluation protocol consists of the following domains: physical and mental health, social and psycho-emotional wellbeing. During the follow-up, we collected demographic and epidemiological characteristics, vaccination status, baseline chronic medical conditions and clinical features during the acute phase of SARS-CoV-2, as well as the clinical symptoms that were persistent at the time of the first visit. We collected this information in a structured questionnaire (which medical staff filled in). For this data analysis, we excluded patients who had not consented to the follow-up visits or who could not be reached, as well as those who showed no symptoms at testing, or those in whom co-infections were diagnosed. Descriptive statistics were used to present the data. The ethics committee of Riga Stradins University and the Institutional Review Board of Children's Clinical University Hospital (No. 6-1/07/35) reviewed and approved the study protocol questionnaire and informed consent forms. 
Table 2 Post-COVID-19 follow-up clinical features

\begin{tabular}{|c|c|c|}
\hline Symptoms & Value & $\%$ \\
\hline Tiredness after sleep & 18 & 20 \\
\hline Persistent fatique & 17 & 18 \\
\hline Loss of taste and/or smell & 15 & 16 \\
\hline Headache & 14 & 15 \\
\hline Sensory disturbances & 10 & 11 \\
\hline Cognitive disturbances & 9 & 10 \\
\hline Prolonged fever & 8 & 9 \\
\hline Stuffy nose & 8 & 9 \\
\hline Joint pain & 7 & 8 \\
\hline Dizziness & 7 & 8 \\
\hline Chest pain & 6 & 7 \\
\hline Cough & 6 & 7 \\
\hline Dry mouth & 6 & 7 \\
\hline Rhinorrhea & 6 & 7 \\
\hline Decreased appetite & 5 & 5 \\
\hline Myalgia & 5 & 5 \\
\hline Orthostatic intolerance & 4 & 4 \\
\hline Sore throat & 4 & 4 \\
\hline Drowsiness & 4 & 4 \\
\hline Red eye syndrome & 3 & 3 \\
\hline Dry eyes & 2 & 2 \\
\hline Shortness of breath & 2 & 2 \\
\hline Sweatiness & 2 & 2 \\
\hline Swollen lymph glands & 2 & 2 \\
\hline Weight loss & 1 & 1 \\
\hline Exercise intolerance & 1 & 1 \\
\hline Microhematuria & 1 & 1 \\
\hline
\end{tabular}

We invited 189 patients who had been tested in an ambulatory setting to participate in the study by telephone, with 92 face-to-face follow-up first visits completed. Among the 189 patients, we excluded 62 from the study who could not be reached, 27 who were not willing to participate in the study and 8 who did not answer the questions about symptoms. No co-infections were observed in patients of the study group. The median age of the study group was 12 years (interquartile range $[\mathrm{IQR}]=8-15$ years), 56 (61\%) were boys. Overall, 18 children reported chronic medical conditions. The median interval from the test to the first follow-up visit was 55 days ( $\mathrm{IQR}=30-104$ days). Among all 92 outpatients during the acute phase of SARS-CoV-2 infection, tiredness was the most commonly reported symptom in $42(46 \%)$ and fever in 39 (42\%), with headaches in 34 (37\%), while a cough was present in $30(33 \%)$ patients. Fever was defined as an axillary temperature of $37.5^{\circ} \mathrm{C}$ or higher. The characteristics of the study population are summarised in Table 1. At the time of the follow-up visit, 45 (49\%) were completely free of any COVID-19related symptoms, while 19\% had 1 symptom, 10\% had 2 , and $22 \%$ had 3 or more. We have not looked at the correlation between the persistence of symptoms and distance from the acute phase of the infection. The Figure shows that patients still commonly reported tiredness (38\%), loss of taste and/or smell (16\%), and headaches (15\%) (Fig. 1). Sensory disturbances (photophobia, sound sensitivity) occurred in 10 (11\%) patients, while cognitive disturbances (memory, attention, and information processing problems) were present in 9 (10\%). Other less common symptoms of the cohort are displayed in Table 2 .

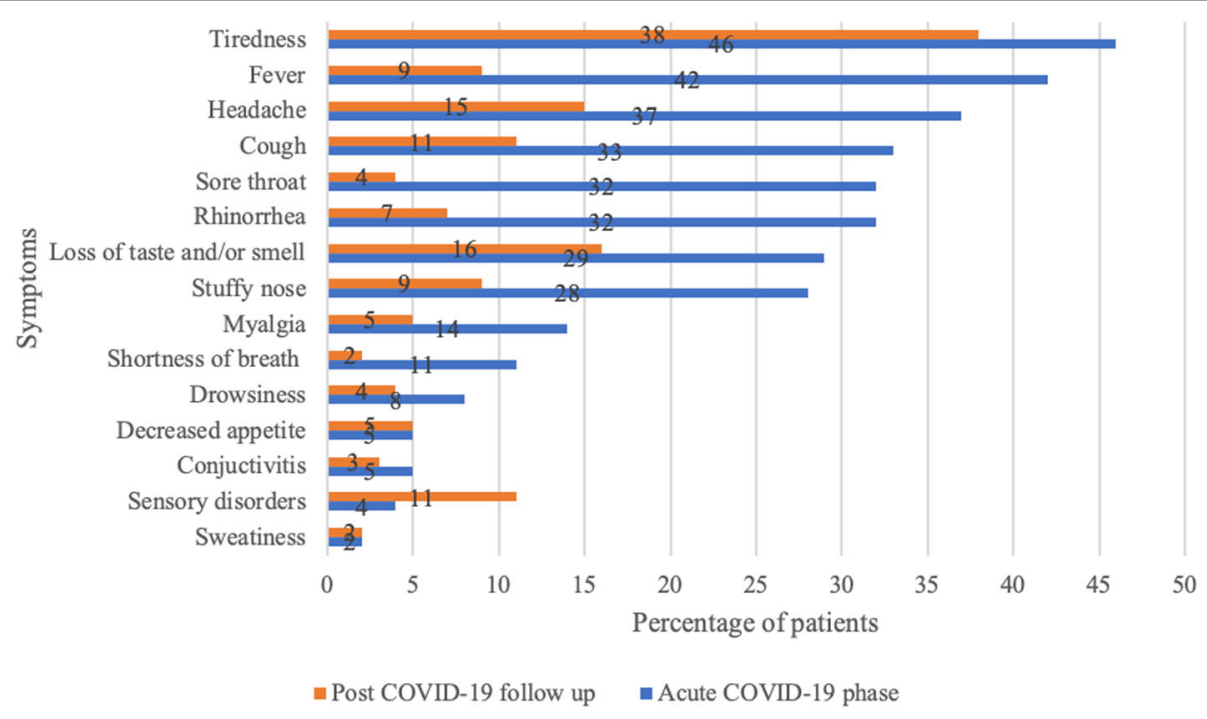

Fig. 1 COVID-19-related symptoms in children during the acute phase of the disease and at the time of the follow-up visit 
To understand the frequency and nature of long-term complications and persistent symptoms, we are continuing our study and following the study design, which consists of outpatient follow-up visits (at 1, 3, 6, 12, and 24 months after COVID-19 onset) at which several domains are evaluated according to a special questionnaire: physical, mental, social and wellbeing. Researchers have shown that even among young adults aged 18-34 years with no chronic medical conditions, nearly one in five reported prolonged COVID-19-related symptoms [3]. The most commonly reported were fatigue, loss of taste and/or smell and headaches $[3,5]$. Our study represents the clinical features of paediatric post-acute COVID-19, with tiredness, loss of taste and/or smell, and headaches among those most commonly reported in outpatients. Previous studies in children found similar presenting symptoms, but they were more common in hospitalized patients $[6,7]$. According to our data, the most common post-acute COVID-19 clinical features were noted in children from 10 to 18 years. In conclusion, future studies assessing organ damage will be needed to better understand and characterise Long COVID in children [11]. This study has several limitations. Firstly, this was a retrospective study. Secondly, this study includes a small sample size of patients. Thirdly, the absence of a control group made a comparison with one impossible.

In conclusion, up to 1-3 months after symptom onset, $51 \%$ of children with COVID-19 had complaints, mainly tiredness, loss of taste/smell and headaches. A prolonged multidisciplinary follow-up of patients with COVID-19 seems relevant, whatever the severity of the symptoms.

\section{Abbreviations}

SARS-CoV-2: Severe acute respiratory syndrome coronavirus 2; COVID-

19: Coronavirus disease 2019

\section{Acknowledgements}

We thank the patients and their families who enrolled in this study during the COVID-19 pandemic.

\section{Authors' contributions}

LS conceptualized and designed the study, designed the data collection instrument, collected data, contributed to the data analysis, interpretation, development of tables and figures, drafted the initial manuscript, and revised the manuscript. IR and ZP conceptualized and designed the study, designed the data collection instrument, collected data, reviewed the collected data, contributed to the data analysis and interpretation, and drafted the initial manuscript and revised the manuscript. JP conceptualized and designed the study, designed the data collection instruments, coordinated and supervised the data collection, and critically reviewed the manuscript for important intellectual content. All authors approved the final manuscript as submitted and agree to be accountable for all aspects of the work.

\section{Funding}

This study was supported by the National Research Program to Mitigate Consequences of COVID-19 (VPP-COVID-2020/1-0011).

\section{Availability of data and materials}

All data generated or analysed during this study are included in this published article.

\section{Declarations}

\section{Ethics approval and consent to participate}

The study protocol questionnaire and informed consent forms were reviewed and approved by the ethics committee of Riga Stradins University and by the Institutional Review Board of Children's Clinical University Hospital (No. 6-1/07/35). In the case of minors, parental consent has been obtained.

\section{Consent for publication}

Not applicable.

\section{Competing interests}

The authors have indicated they have no potential conflicts of interest to disclose.

Received: 6 January 2021 Accepted: 4 August 2021

Published online: 26 August 2021

References

1. COVID Symptom Study. How long does COVID-19 last? Kings College London, 2020. https://covid.joinzoe.com/post/covid-long-term?fbclid=IwAR1 RxlcmmdL-EFjh_al-.

2. Carfi A, Bernabei R, Landi F. Gemelli against COVID-19 post-acute care study group. Persistent symptoms in patients after acute COVID-19. JAMA. 2020; 324(6):603-5. https://doi.org/10.1001/jama.2020.12603.

3. Tenforde MW, Kim SS, Lindsell CJ, Rose Billig E, Shapiro NI, Files DK, et al. Symptom Duration and Risk Factors for Delayed Return to Usual Health Among Outpatients with COVID-19 in a Multistate Health Care Systems Network - United States, March-June 2020. MMWR Morb Mortal Wkly Rep. 2020;69(30):993-8. https://doi.org/10.15585/mmwr.mm6930e1.

4. Carvalho-Schneider C, Laurent E, Lemaignen A, Beaufils E, Bourbao-Tournois C, Laribi S, et al. Follow-up of adults with noncritical COVID-19 two months after symptom onset. Clin Microbiol Infect. 2020;\$1198-743X(20):30606. https://doi.org/10.1016/j.cmi.2020.09.052.

5. Nehme M, Braillard O, Alcoba G, Aebischer Perone S, Courvoisier D, Chappuis F, et al. COVID-19 symptoms: longitudinal evolution and persistence in outpatient settings. Ann Intern Med. 2020;174(5):723-5. https://doi.org/10.7326/M20-5926.

6. Buonsenso D, Munblit D, De Rose C, Sinatti D, Ricchiuto A, Carfi A, et al. Preliminary evidence on long COVID in children. Acta Paediatr. 2021;110(7): 2208-11. https://doi.org/10.1111/apa.15870.

7. Osmanov IM, Spiridonova E, Bobkova P, Gamirova A, Shikhaleva A, Andreeva $M$, et al. Risk factors for long covid in previously hospitalised children using the ISARIC global follow-up protocol: a prospective cohort study. Eur Respir J. 2021;1:2101341. https://doi.org/10.1183/13993003.01341-2021.

8. Hoang A, Chorath K, Moreira A, Evans M, Burmeister-Mortonet F, Burmeister F, et al. COVID-19 in 7780 pediatric patients: a systematic review. EClinicalMedicine. 2020;24:100433. https://doi.org/10.1016/j. eclinm.2020.100433.

9. Halpin SJ, Mclvor C, Whyatt G, Adams A, Harvey O, McLean L, et al. Postdischarge symptoms and rehabilitation needs in survivors of COVID-19 infection: a cross-sectional evaluation. J Med Virol. 2021;93(2):1013-22. https://doi.org/10.1002/jmv.26368.

10. Greenhalgh T, Knight M, A'Court C, Buxton M, Husain L. Management of post-acute covid-19 in primary care. BMJ. 2020;370:m3026. https://doi.org/1 $0.1136 / \mathrm{bmj} . \mathrm{m} 3026$.

11. Buonsenso D, Di Giuda D, Sigfrid L, Pizzuto DA, Di Sante G, et al. Evidence of lung perfusion defects and ongoing inflammation in an adolescent with post-acute sequelae of SARS-CoV-2 infection. Lancet Child Adolesc Health. 2021. https://doi.org/10.1016/S2352-4642(21)00196-6.

\section{Publisher's Note}

Springer Nature remains neutral with regard to jurisdictional claims in published maps and institutional affiliations. 